\title{
INATIVIDADE FÍSICA NO LAZER EM ESTUDANTES DE EDUCAÇÃO FÍSICA, BAHIA
}

\author{
LEISURE-TIME PHYSICAL INACTIVITY IN UNDERGRADUATE STUDENTS OF \\ PHYSICAL EDUCATION FROM BAHIA, BRAZIL
}

\section{Dayana Chaves Franco ${ }^{a}$, Thiago Ferreira de Sousa ${ }^{b}$}

\author{
adayanafranco18@gmail.com, btfsousa_thiago@yahoo.com.br \\ Universidade Federal do Triângulo Mineiro - Uberaba (MG), Brasil
}

Data de recebimento do artigo: 31/05/2017

Data de aceite do artigo: 01/08/2017

\section{RESUMO}

Introdução: A inatividade física é um dos principais fatores de risco relacionados à mortalidade mundial, com grandes implicaçôes em doenças crônicas não transmissíveis. Em relação aos universitários, a inatividade física representa um comportamento prevalente. Objetivo: estimar a prevalência e os fatores associados à inatividade física no lazer em universitários de um curso de educação física. Metodologia: trata-se de um estudo de caráter transversal, realizado em uma instituiçáo de ensino superior pública do estado da Bahia, com os universitários do curso de educação física. A inatividade física no lazer é o desfecho investigado e a medida de associaçáo foram as Razôes de Prevalências (RP). O nível de significância adotado foi de 5\%. Resultados: Participaram deste estudo 111 estudantes. A prevalência de universitários inativos no lazer foi de $14,4 \%$. O sexo feminino associou-se à inatividade física no lazer (RP: 6,22; IC95\%: 1,62 - 23,90). Além disso, os universitários de maior faixa etária foram menos inativos quando comparados aos mais jovens (RP: 0,18; IC95\%: 0,04-0,92), e os universitários casados foram mais inativos. Conclusáo: A prevalência de inatividade física no lazer dos estudantes de Educação Física foi baixa. Os universitários do sexo feminino com idade entre 18 e 20 anos e casados tiveram maiores prevalências de inatividade física no lazer.

Palavras-chave: Comportamento de risco; estudantes; educação física; atividades de lazer.

\section{ABSTRACT}

Introduction: Physical inactivity is one of the main risk factors related to worldwide mortality, with great implications for chronic diseases. In relation to university students, physical inactivity represents a prevalent behavior. Objective: To estimate the prevalence and factors associated with leisure-time physical inactivity in university students of a Physical Education course. Methodology: It is a cross-sectional study carried out in a public higher education institution from state of Bahia. The physical inactivity investigated by Prevalence Ratio (PR). Significance level was 5\%. Results: 111 university students participated in it. The prevalence of physical inactivity in leisure-time was $14.4 \%$. Female students were associated with leisure-time physical inactivity (PR: 6.22; CI95\%: $1.62-23.90$ ), university students of higher age groups were less inactive in leisure-time when compared to younger ones (PR: 0.18; 95\%CI: $0.04-0.92$ ), and married students were more inactive in leisure-time. Conclusion: The prevalence of physical inactivity in the leisure-time was lower in Physical Education students. Married female students aged between 18 to 20 years old had higher prevalence of leisure-time physical inactivity.

Keywords: Risk behavior; students; physical education; leisure activities. 


\section{Introdução}

A inatividade física (IF) é um dos principais fatores de risco relacionados à mortalidade mundial, com grandes implicaçôes em doenças crônicas não transmissíveis ${ }^{1}$. Apesar das evidências sobre os seus prejuízos à saúde, observa-se uma pandemia de $\mathrm{IF}^{2}$, que contribui diretamente com os gastos em saúde pública ${ }^{3}$. As prevalências de IF na população brasileira são elevadas, em torno de $30,3 \%$ a $58 \%$ em diferentes estudos ${ }^{4-7}$, tanto no lazer ${ }^{5}$ quanto entre todos os domínios ${ }^{4,6,7}$. Esses dados são preocupantes, uma vez que, de acordo com a Organização Mundial da Saúde (OMS), até 2030, as doenças cardiovasculares deverão ocupar o primeiro lugar em causas de mortes no mundo ${ }^{8}$.

Em relação aos universitários, população que cresceu no Brasil, a IF representa um comportamento com proporçóes que variam entre 0 e 78,9\% em diferentes regiôes ${ }^{9}$. Em universitários do curso de educação física (EF), a literatura mostra menores prevalências de $\mathrm{IF}^{10-12}$. Entre os principais fatores associados à IF no lazer, estáo sexo feminino ${ }^{10-12}$, idade mais avançada ${ }^{11}$, menor nível socioeconômico $^{10,11}$ e casamento ${ }^{12}$.

É importante que os alunos do curso de EF apresentem menores prevalências desse comportamento de risco, pois eles podem favorecer a prática profissional mediante a promoçáo de um estilo de vida ativo para a população, tendo em vista o conhecimento acadêmico e a experiência prática que possuem. Diante disso, a compreensão sobre o perfil de IF no lazer desses profissionais torna-se essencial. Este estudo teve como objetivo estimar a prevalência e os fatores associados à inatividade física no lazer em universitários de um curso de educação física.

\section{Metodologia}

Este estudo é de característica transversal, realizado em uma instituição de ensino superior pública, de campus único, do estado da Bahia. A pesquisa foi aprovada pelo Comitê de Ética em Pesquisa (CEP) local, sob número de protocolo 343/09. Os universitários que participaram assinaram o Termo de Consentimento Livre e Esclarecido e foram informados sobre o objetivo do estudo.

A população deste estudo foi composta pelos universitários do curso de EF da universidade, regularmente matriculados no segundo semestre do ano de 2014 , tendo como base a lista dos estudantes matriculados no referido semestre, obtida no colegiado do curso, perfazendo 131 universitários. Realizou-se um censo no curso de EF, e não fizeram parte da população do estudo os alunos com matrícula especial ou ouvintes. A coleta de dados ocorreu nos meses de setembro e outubro do ano de 2014, nas dependências da universidade, durante os intervalos das aulas. Os estudantes foram convidados pessoalmente e participaram do estudo de forma individual ou em pequenos grupos. As informaçóes foram obtidas por meio do questionário empregado por Sousa et al. ${ }^{13} \mathrm{em}$ pesquisa anterior com estudantes de EF.

A IF no lazer foi a categoria investigada neste estudo. Foram considerados inativos no lazer os estudantes que responderam não ter praticado em nenhum dia, nas duas últimas semanas, atividades físicas no lazer. As variáveis independentes foram: sexo (masculino e feminino), faixa etária em anos completos (18-20, 21$24, \geq 25$ ) situação conjugal (solteiro e casado), ano de entrada no curso $(2014,2013,2012$ e 2011 ou anterior), consumo de frutas/sucos ( $\geq 5$ dias por semana e $\leq 4$ dias por semana) $)^{14}$, consumo de verduras/saladas ( $\geq 5$ dias por semana e $\leq 4$ dias por semana) ${ }^{14}$ e índice de massa corporal (IMC), calculado com base na equação padrão (massa corporal dividida pela estatura ao quadrado), mediante as informaçóes referidas da massa corporal e estatura, classificado em peso normal (IMC: 18,5 a $24,9 \mathrm{~kg} / \mathrm{m}^{2}$ ) e excesso de peso (IMC: $\left.\geq 25,0 \mathrm{~kg} / \mathrm{m}^{2}\right)^{15}$. Os universitários com baixo peso (IMC: $\leq 18,4 \mathrm{~kg} / \mathrm{m}^{2}$ ) foram excluídos devido à baixa frequência $(n=4 ; 3,7 \%)$.

Os dados foram tabulados na versão 2007 do software Excel, e as análises estatísticas na versão 15.0 do programa SPSS. Utilizou-se a estatística descritiva das frequências absolutas e relativas, média e desvio padrão, mínimo e máximo. As Razões de Prevalências (RP), estimadas pela regressão de Poisson, com ajuste para a variância robusta nas análises brutas e ajustadas, foram utilizadas como medida de associação. Todas as variáveis independentes foram para a análise ajustada. $\mathrm{O}$ nível de significância adotado foi de $5 \%$.

\section{Resultados}

Participaram deste estudo 111 estudantes do curso de EF. A média etária foi de 24,7 anos (dp: 6,6 anos; 18 a 46 anos). A descrição sociodemográfica e de vínculo com a universidade é apresentada na Tabela 1. A maior parte dos universitários foi do sexo masculino $(69,4 \%)$ e solteiros $(83,8 \%)$. Predominaram os universitários com idade igual ou superior a 25 anos $(32,4 \%)$ e ingresso na universidade em 2011 ou em anos anteriores (29,7\%). A maior parte relatou consumir frutas em cinco ou mais dias por semana $(51,4 \%)$ e verduras/saladas em cinco ou mais dias por semana $(52,3 \%)$. 
Tabela 1: Caracterização da amostra de universitários de educação física. Bahia. 2014.

\begin{tabular}{|c|c|c|}
\hline Variáveis & $\mathbf{N}$ & $\%$ \\
\hline \multicolumn{3}{|l|}{ Sexo } \\
\hline Masculino & 77 & 69,4 \\
\hline Feminino & 34 & 30,6 \\
\hline \multicolumn{3}{|l|}{ Faixa etária } \\
\hline 18 a 20 anos & 34 & 32,4 \\
\hline 21 a 24 anos & 35 & 33,3 \\
\hline$\geq 25$ anos & 36 & 34,3 \\
\hline \multicolumn{3}{|l|}{ Situação conjugal } \\
\hline Solteiro & 93 & 84,5 \\
\hline Casado & 17 & 15,5 \\
\hline \multicolumn{3}{|l|}{ Anos de estudo no curso } \\
\hline 2014 & 31 & 27,9 \\
\hline 2013 & 22 & 19,8 \\
\hline 2012 & 25 & 22,5 \\
\hline $2011 \mathrm{ou}$ anteriores & 33 & 29,8 \\
\hline \multicolumn{3}{|l|}{ Consumo de frutas/sucos } \\
\hline$\geq 5$ dias por semana & 57 & 52,3 \\
\hline$\leq 4$ dias por semana & 52 & 47,7 \\
\hline \multicolumn{3}{|c|}{ Consumo de verduras/saladas } \\
\hline$\geq 5$ dias por semana & 58 & 53,2 \\
\hline$\leq 4$ dias por semana & 51 & 46,8 \\
\hline \multicolumn{3}{|l|}{ Índice de massa corporal } \\
\hline Peso normal & 69 & 66,3 \\
\hline Excesso de peso corporal & 35 & 33,7 \\
\hline
\end{tabular}

\%: Proporção.

Fonte: Elaboração dos autores (2017).

A prevalência de universitários inativos no lazer foi de 14,4\%. A Tabela 2 apresenta a análise bruta e multivariada entre as variáveis exploratórias e a IF no lazer. $\mathrm{Na}$ análise bruta, as mulheres foram significativamente mais inativas no lazer do que os homens $(\mathrm{RP}=6,79$; IC95\%=2,36-19,55). $\mathrm{Na}$ análise ajustada, o sexo feminino associou-se à IF no lazer $(\mathrm{RP}=6,22$; IC95\%=1,62; 23,90). Além disso, os universitários de maior faixa etária foram menos inativos no lazer quando comparados aos mais jovens $(R P=0,18$; IC95\% $\%, 04-0,92)$ e os universitários casados foram mais inativos no lazer.

Tabela 2: Associação entre variáveis sociodemográficas, de vínculo com a universidade, hábitos alimentares e índice de massa corporal com a inatividade física no lazer em estudantes de educação física. Bahia. 2014.Discussão

\begin{tabular}{|c|c|c|c|c|c|c|}
\hline \multirow[b]{2}{*}{ Variáveis } & \multicolumn{6}{|c|}{ Inatividade física no lazer } \\
\hline & $\mathbf{N}$ & $\%$ & $\begin{array}{l}\text { RP bruta } \\
\text { (IC95\%) }\end{array}$ & $\mathbf{p}$ & $\begin{array}{l}\text { RP ajustada* } \\
\text { (IC95\%) }\end{array}$ & $\mathbf{p}$ \\
\hline Sexo & & & & $<0,01$ & & $<0,01$ \\
\hline Masculino & 77 & 5,2 & 1,00 & & 1,00 & \\
\hline Feminino & 34 & 35,3 & $6,79(2,36 ; 19,55)$ & & $6,22(1,62 ; 23,90)$ & \\
\hline Faixa etária & & & & $0,18^{* *}$ & & $0,03^{* *}$ \\
\hline 18 a 20 anos & 34 & 23,5 & 1,00 & & 1,00 & \\
\hline 21 a 24 anos & 35 & 8,6 & $0,36(0,10 ; 1,26)$ & & $0,23(0,03 ; 1,95)$ & \\
\hline$\geq 25$ anos & 36 & 11,1 & $0,47(0,16 ; 1,43)$ & & $0,18(0,04 ; 0,92)$ & \\
\hline Situação conjugal & & & & 0,29 & & $<0,01$ \\
\hline Solteiro & 93 & 12,9 & 1,00 & & 1,00 & \\
\hline Casado & 17 & 23,5 & $1,82(0,58 ; 5,65)$ & & $4,62(1,61 ; 13,25)$ & \\
\hline Anos de estudo no curso & & & & $0,19^{* *}$ & & $0,99^{* *}$ \\
\hline
\end{tabular}


Tabela 2: Continuação.

\begin{tabular}{|c|c|c|c|c|c|c|}
\hline \multirow[b]{2}{*}{ Variáveis } & \multicolumn{6}{|c|}{ Inatividade física no lazer } \\
\hline & $\mathbf{N}$ & $\%$ & $\begin{array}{l}\text { RP bruta } \\
\text { (IC95\%) }\end{array}$ & $\mathbf{p}$ & $\begin{array}{l}\text { RP ajustada* } \\
\text { (IC95\%) }\end{array}$ & $\mathbf{p}$ \\
\hline 2014 & 31 & 19,4 & 1,00 & & 1,00 & \\
\hline 2013 & 22 & 18,2 & $0,94(0,30 ; 2,94)$ & & $0,54(0,14 ; 2,14)$ & \\
\hline 2012 & 25 & 12,0 & $0,62(0,17 ; 2,23)$ & & $0,52(0,14 ; 2.01)$ & \\
\hline $2011 \mathrm{ou}$ anteriores & 33 & 9,1 & $0,47(0,29 ; 1,72)$ & & & \\
\hline Consumo de frutas/sucos & & & & 0,73 & & 0,78 \\
\hline$\geq 5$ dias por semana & 57 & 15,8 & 1,00 & & 1,00 & \\
\hline$\leq 4$ dias por semana & 52 & 13,5 & $0,85(0,34 ; 2,12)$ & & $1,17(0,42 ; 3,25)$ & \\
\hline Consumo de verduras/saladas & & & & 0,19 & & 0,60 \\
\hline$\geq 5$ dias por semana & 58 & 19,0 & 1,00 & & 1,00 & \\
\hline$\leq 4$ dias por semana & 51 & 9,8 & $0,52(0,19 ; 1,39)$ & & $0,69(0,17 ; 2,83)$ & \\
\hline Índice de massa corporal & & & & 0,98 & & 0,75 \\
\hline Peso normal & 69 & 14,5 & 1,00 & & 1,00 & \\
\hline Excesso de peso corporal & 35 & 14,3 & $0,99(0,36 ; 2,66)$ & & $1,17(0,45 ; 3,05)$ & \\
\hline
\end{tabular}

\%=Prevalência; RP=Razóes de Prevalências; IC95\%=Intervalo de Confiança a 95\%; *Ajustada para todas as variáveis; **p valor do teste de Wald para tendência linear. Fonte: Elaboração dos autores (2017).

Neste estudo, a prevalência de IF no lazer entre os universitários foi baixa. Observou-se que as mulheres e os universitários casados foram associados com maiores prevalências de IF no lazer, por outro lado, os universitários da maior faixa etária apresentaram menor prevalência.

A prevalência de IF no lazer entre os universitários de EF foi baixa quando comparada com os universitários dos outros cursos de graduação das áreas de saúde, exatas, humanas, agrárias, linguagens e biológicas $^{11,12,16-18}$. Além disso, a prevalência deste estudo foi menor que a de outras pesquisas com universitários do curso de $\mathrm{EF}^{10,11,13}$. As menores prevalências de IF para os universitários de EF podem estar relacionadas ao perfil comportamental desse grupo, com maior envolvimento em atividades físicas no lazer antes do ingresso na universidade ${ }^{13}$

Do mesmo modo que na população em geral ${ }^{19,20}$, as universitárias deste estudo foram mais inativas no lazer, assim como em outros estudos com universitários ${ }^{10-12,21}$. A menor possibilidade de IF no lazer em homens pode ocorrer devido aos motivos culturais que favorecem a prática, pois esse grupo tende a praticar por diversão $\mathrm{o}^{22} \mathrm{e}$ apresenta maior apoio social e familiar para a realizaçáo de atividades físicas ${ }^{23}$.

Em relação à faixa etária, observou-se que, com o aumento da idade, os estudantes foram menos inativos no lazer, o que se opóe a outros estudos com universitários, que observaram menores prevalências de IF nos mais jovens ${ }^{10-12,18}$. O resultado encontrado entre os universitários casados e a IF no lazer foi semelhante ao estudo de Santos et al., realizado com estudantes de uma universidade na cidade de Pelotas $/ \mathrm{RS}^{12}$, e pode estar relacionado com questóes voltadas às obrigaçóes familiares e aos estudos, portanto, estes participantes podem estar ocupando o tempo livre com atividades não relacionadas ao lazer ativo. A diminuição da participação em açóes esportivas com amigos e o menor desejo de prática de atividades físicas no lazer para fins estéticos podem, também, favorecer a maior IF para aqueles com companheiro.

Uma das limitaçóes deste estudo é o delineamento transversal, que inviabiliza relaçóes de causalidade, pois viola o princípio da temporalidade. Além disso, o envolvimento de universitários de uma única instituição pública pode não representar o perfil de estudantes das instituições particulares, já que estes estudantes podem apresentar perfil socioeconômico diferenciado. Soma-se a isso o uso de um questionário para a mensuração de informações sobre comportamentos, em virtude do viés recordatório e da superestimação da prática. $\mathrm{O}$ uso de medidas referidas da massa corporal e estatura para o cálculo do IMC pode representar viés de mensuração, contudo, estudo prévio apontou níveis satisfatórios para o emprego com universitários ${ }^{24}$.

\section{Conclusão}

A prevalência de IF no lazer dos estudantes de EF foi baixa. Os universitários do sexo feminino, aqueles de idade entre 18 e 20 anos e os casados tiveram maiores prevalências de IF no lazer. A compreensão sobre o perfil epidemiológico da IF em universitários é essencial para a formulação de açôes que possam favorecer a manutenção da prática durante o período universitário. 


\section{Referências}

1. World Health Organization. Global recommendations on physical activity for health. Geneva: WHO; 2010. 58 p.

2. Kohl HW, Craig CL, Lambert EV, Inoue S, Alkandari JR, Leetongin $\mathrm{G}$, et al. The pandemic of physical inactivity: global action for public health. Lancet. 2012;380(9838):294-305.

3. Bueno DR, Marucci MFN, Codogno JS, Roediger MA. Os custos da inatividade física no mundo: estudo de revisão. Cien Saude Colet. 2016;21(4):1001-10.

4. Hallal PC, Victora CG, Wells JCK, Lima RC. Physical inactivity: prevalence and associated variables in Brazilian adults. Med Sci Sports Exerc. 2003;35(11):1894-900.

5. Brasil. Ministério da Saúde. Secretaria de Vigilância em Saúde. Secretaria de Gestão Estratégica e Participativa. Vigitel Brasil 2012: vigilância de fatores de risco e proteção para doenças crônicas por inquérito. Brasília, DF: Ministério da Saúde; 2012.

6. Siqueira FV, Facchini LA, Piccini RX, Tomasi E, Thumé E, Silveira DS, et al. Atividade física em adultos e idosos residentes em áreas de abrangência de unidades básicas de saúde de municípios das regióes Sul e Nordeste do Brasil. Cad Saude Publica. 2008;24(1):39-54.

7. Alves JGB, Siqueira FV, Figueiroa JN, Facchini LA, Silveira DS, Piccini RX, et al. Prevalência de adultos e idosos insuficientemente ativos moradores em áreas de unidades básicas de saúde com e sem Programa Saúde da Família em Pernambuco, Brasil. Cad Saude Publica. 2010;26(3):543-56.

8. World Health Organization. World health statistics 2008. Geneva: WHO; 2008. 110 p.

9. Sousa TF. Inatividade física em universitários brasileiros: uma revisão sistemática. Rev Bras Ciên Saúde. 2011;29(9):47-55.

10. Bielemann RM, Karine G, Azevedo MR, Reichert FF. Prática de atividade física no lazer entre acadêmicos de educação física e fatores associados. Rev Bras Ativ Fís Saúde. 2007;12:(3)65-72.

11. Mielke GI, Ramis TR, Habeyche EC, Oliz MM, Tessmer MGS, Azevedo MR, et al. Atividade física e fatores associados em universitários do primeiro ano da Universidade Federal de Pelotas. Rev Bras Ativ Fís Saúde. 2010;15(1):57-64.

12. Santos TS, Leão OAA, Leite JS, Silva MC. Atividade física em acadêmicos de educação física: um estudo longitudinal. Rev Bras Ativ Fís Saúde. 2017;22(1):76-84.
13. Sousa TF, Santos SFS, Pie ACS, Rossato LC. Associação entre indicadores de prática de atividades físicas na adolescência com o nível atual de prática de atividades físicas no lazer em acadêmicos de um curso de educação física no Nordeste do Brasil. Pensar Prát. 2009;12(3):1-12.

14. World Health Organization. World Health Report 2002: reducing risks, promoting healthy life. Geneva: WHO; 2002. $250 \mathrm{p}$.

15. World Health Organization. Obesity: preventing and managing the global epidemic: report of a WHO consulation of obesity. Geneva: WHO; 1997. 252p.

16. Resende MA, Resende RBV, Tavares RS, Santos CRR, Barreto-Filho JAS. Estudo comparativo do perfil pró-aterosclerótico de estudantes de medicina e educação física. Arq Bras Cardiol. 2010;95(1):1-9.

17. Santos SFS, Freitas Junior IF, Alvarenga AM, Fonseca SA, Virtuoso Junior JS, Sousa TF. Prevalence and factors associated with leisure-time physical activity: survey repeated in university students. Rev Bras Cineantropom Hum. 2016;18(5):577-90.

18. Sousa TF, José HPM, Barbosa AR. Condutas negativas à saúde em estudantes universitários brasileiros. Cienc Saude Colet. 2013;18(12):3563-75.

19. Del Duca GF, Nahas MV, Garcia LMT, Peres MA. Como gênero e escolaridade interagem nos padróes de inatividade física em diferentes domínios em adultos? Rev. Bras. Educ. Fís. Esporte. 2015;29(4):653-61.

20. Pazin J, Duarte MFS, Borgatto AF, Peres MA, Poeta LS. Atividade física no lazer, deslocamento, apoio social e percepção do ambiente urbano em homens e mulheres de Florianópolis/SC. Rev. Bras. Educ. Fís. Esporte. 2016;30(3):743-55.

21. Palma A, Abreu RA, Cunha CA. Comportamento de risco e vulnerabilidade entre estudantes de educaçáo física. Rev Bras Epidemiol. 2007;10(1):117-26.

22. Monteiro CA, Conde WL, Matsudo SM, Matsudo VR. Bonseñor IM, Lotufo PA. A descriptive epidemiology of leisure-time physical activity in Brazil, 1996-1997. Rev Panam Salud Publica. 2003;14(4):246-54.

23. Gonçalves H, Hallal PC, Amorim TC, Araújo CLP, Menezes AMB. Fatores socioculturais e nível de atividade física no início da adolescência. Rev Panam Salud Publica. 2007;22(4):256-53.

24. Sousa TF, Barbosa AR. Validade das medidas referidas da massa corporal e estatura em universitários. ABCS Health Sci. 2016;41(2):71-7.

\section{Como citar este artigo:}

Franco DC, Sousa TF. Inatividade física no lazer em estudantes de educação física, Bahia. Rev. Aten. Saúde. 2017;15(53)76-80. 